\title{
Rehabilitación para el trabajo: perspectivas ante una nueva pandemia COVID-19
}

\author{
Vocational rehabilitation: prospects for a new COVID-19 pandemic
}

\author{
Dr. Ignacio Devesa Gutiérrez
}

Tniciamos el año 2020 enfrentando un nuevo L paradigma ante las situaciones causadas por la pandemia del COVID-19, que nos desafió a una nueva realidad caracterizada por el confinamiento social y la incertidumbre ante una enfermedad emergente, la que nos orilló a cambiar las condiciones de convivencia social y que afectó gravemente la rutina de las instalaciones hospitalarias, obligando a la conversión de actividades hacia hospitales COVID. Esto trajo como consecuencia que la atención a las personas con discapacidad se viera afectada, lo cual representa nuevos retos en la medicina de rehabilitación que se tiene que adecuar a la nueva realidad.

La infección por COVID no sólo afecta al sistema cardiorrespiratorio, sino también al nervioso y musculoesquelético, entre otras patologías. Produce secuelas que alteran las actividades de la vida diaria humana, incluyendo, por supuesto, las actividades escolares y laborales.

Las secuelas producidas por el COVID son múltiples y diversas, dejando consecuencias secundarias al reposo prolongado, síndrome de desgaste post covid y al uso de intubación respiratoria por tiempo prolongado que traen como resultado cansancio extremo, disnea de pequeños y medianos esfuerzos, debilidad muscular que complican el retorno al trabajo de los afectados. Además, se han reportado alteraciones que son susceptibles de recibir tratamiento de rehabilitación, principalmente el desacondicionamiento cardiopulmonar, trastornos de la deglución y del habla secundarios a la intubación, trastornos neurológicos como neuropatía, polirradiculoneuropatías, mielopatía, encefalopatía, enfermedad vascular cerebral, amputación de extremidades, problemas cognitivos, psicológicos por estrés postraumático y sus alteraciones en la esfera social, tanto individual como familiar y de grupo. Todo esto sin considerar las comorbilidades como la diabetes mellitus, la obesidad y la hipertensión arterial que se ven gravemente alteradas. Estamos también ante una entidad nueva llamada síndrome postCOVID, que se caracteriza por cansancio físico extremo, disnea, tos acompañada de cefalea, dolor muscular y articular, pérdida del olfato o del gusto, trastornos de la memoria, concentración y del sueño, que pueden estar presentes no sólo en personas que sufrieron un serio problema por el COVID, sino también en aquellos que cursaron con cuadros leves. Asimismo, en estos días también nos enfrentamos a la expectativa de los resultados de los programas de vacunación, donde se han reportado algún tipo de reacciones secundarias, en especial en pacientes con antecedentes alérgicos.

Los grupos de edad más afectados en México son los que van de los 25 a los 60 años, que es la población considerada como económicamente activa, aunque se ha demostrado que un porcentaje de hasta $70 \%$ de los infectados cursarán con síntomas mínimos a moderados o serán asintomáticos.
Mexicana de Medicina Física y Rehabilitación.
Recibido:

enero, 2021.

Aceptado:

febrero, 2021.
Citar como: Devesa GI. Rehabilitación para el trabajo: perspectivas ante una nueva pandemia COVID-19. Rev Mex Med Fis Rehab. 2020; 32 (1-2): 4-5. https://dx.doi.org/10.35366/98512 
Las tasas de mortalidad se encuentran principalmente en los adultos mayores y también en pacientes en edad productiva.

Uno de los objetivos de la Medicina Física y Rehabilitación es la culminación del proceso rehabilitatorio de las personas con discapacidad para integrarlos a su ámbito social. En muchos casos, pero sobre todo a nivel hospitalario, se ha considerado la infección por COVID como un riesgo del trabajo. Una vez que el paciente se ha recuperado de la infección por COVID-19 y se da por terminado el proceso de rehabilitación habiéndose ya establecido las limitaciones funcionales con las que el paciente se quedó, se debe iniciar el proceso de rehabilitación para el trabajo. El médico especialista en Medicina Física y Rehabilitación es el líder del equipo interdisciplinario y el encargado de elaborar la valoración de la capacidad funcional del paciente. La evaluación debe incluir historia médica completa, con antecedentes laborales del paciente como nombre de la empresa, perfil del puesto de trabajo y características, antigüedad, salario, tiempo de incapacidad, clasificación del padecimiento desde el punto de vista laboral, horario de trabajo, forma y tiempo de traslado de la casa a la empresa, grado de estudios, expectativas laborales del paciente, entre otros. Se requiere completar con un examen médico completo y funcional que incluya una evaluación cardiorrespiratoria y neurológica que determine la existencia o no de secuelas o síndrome post-COVID. No hay que omitir una evaluación psicológica del área cognitiva en búsqueda de disfunción intelectual, síndrome postraumático, entre otros, sin olvidar la evaluación de la familia y su núcleo social. Una vez definido el diagnóstico, es necesario establecer el pronóstico funcional que exprese las limitaciones que tiene el individuo, así como sus potencialidades para, posteriormente, elaborar la confrontación del padecimiento con el puesto de trabajo y establecer un plan de evaluación de sus capacidades funcionales. Es en este momento que el médico especialista en Medicina Física y Rehabilitación refiere al paciente al equipo interdisciplinario de rehabilitación, integrado por Gestoría Ocupacional para afinar los detalles del análisis de puesto, así como realizar visitas de concientización a las empresas. El Servicio de Psicología realizará el diagnóstico y tratamiento psicológico mediante la aplicación de pruebas dirigidas a la determinación de las aptitudes del paciente, tests enfocados a establecer la presencia de daño cerebral y ganancia secundaria, al igual que psicoterapia individual, grupal o familiar. La evaluación funcional del paciente se lleva a cabo en las áreas de evaluación funcional, mediante diversos procedimientos que van desde los tests computarizados de simulación laboral, hasta la reproducción de las actividades que se efectúan en el trabajo en talleres y áreas de terapia ocupacional, como es el caso del Taller de Adiestramiento en Actividades Múltiples, para el manejo de máquinas y herramienta, donde se efectúa la evaluación y capacitación laboral, reforzamiento de actitudes positivas hacia el trabajo y actividades de simulación y reentrenamiento laboral, elaboración de ayudas y aditamentos, así como adaptaciones para realizar las actividades del puesto especifico. En estas áreas también se lleva a cabo la detección de habilidades y destrezas, análisis de tiempos y movimientos y realiza pruebas en equipos computarizados de evaluación funcional para el trabajo. En aquellos casos en que el padecimiento del paciente no es compatible con el puesto de trabajo que realizaba el paciente, se recomienda valorar la posibilidad de una reubicación laboral en la misma empresa; cuando no existe esta posibilidad, puede ser capacitado en otro tipo de actividad, llegando incluso a la reconversión de habilidades para que ocupe otro puesto laboral.

La reconversión de actividades puede realizarse en Centros de Capacitación y Rehabilitación para el trabajo (CECART), o en los diversos centros de capacitación para el trabajo a nivel nacional y a través de la Red Nacional de Vinculación Laboral de la Secretaría del Trabajo y Previsión Social. El CECART proporciona en forma gratuita a población abierta con discapacidad capacitación y reconversión de actividades en diversos talleres, cuenta con el Programa de Oportunidades para el Empleo a través de la Tecnología en las Américas (POETA), que brinda capacitación en tecnologías de la Información y Comunicación y en Tecnología Adaptada para el empleo y generación de ingresos para personas con discapacidad, talleres de computación, electrónica y electricidad, carpintería y manufactura, entre otros. Una vez que el trabajador es capacitado, pasa a la fase de reacomodo laboral y colocación selectiva. Posteriormente, se realizan visitas a las empresas para hacer seguimiento de los progresos y permanencia en el puesto de trabajo de las personas previamente integradas.

Aún quedan muchas incertidumbres, no sabemos la duración de esta pandemia ni las repercusiones a corto y mediano plazo sobre el empleo; sin embargo, tenemos que adaptarnos a esta nueva normalidad y hacer uso de las experiencias que nos ha dado, seguir manteniendo el uso de los equipos de protección personal, y guardar las distancias recomendadas.

\footnotetext{
Correspondencia:

Dr. Ignacio Devesa Gutiérrez

E-mail: idevesa@yahoo.com
} 